\title{
microRNA-155 regulates angiotensin II type 1 receptor expression in umbilical vein endothelial cells from severely pre-eclamptic pregnant women
}

\author{
WEIWEI CHENG ${ }^{1}$, TE LIU ${ }^{1,2}$, FEIZHOU JIANG ${ }^{1}$, CHUNMIN LIU $^{1}$, XINRONG ZHAO ${ }^{1}$, \\ YONGTAO GAO ${ }^{1}$, HUI WANG ${ }^{1}$ and ZHIXUE LIU ${ }^{3}$ \\ ${ }^{1}$ Central Laboratory, International Peace Maternity and Child Health Hospital, Shanghai Jiaotong University, \\ Shanghai 200030; ${ }^{2}$ School of Environmental Science and Engineering, Donghua University, Shanghai \\ 201620; ${ }^{3}$ School of Life Science and Technology, Tongji University, Shanghai 200092, P.R. China
}

Received October 14, 2010; Accepted December 17, 2010

DOI: $10.3892 /$ ijmm.2011.598

\begin{abstract}
Angiotensin II is critical in pre-eclampsia pathogenesis. In addition, microRNA-155 regulates angiotensin II type 1 receptor expression. We have explored the function of microRNA-155 in pre-eclamptic pregnant women. Human umbilical vein endothelial cells were isolated and cultured from healthy puerperant women and pre-eclampsia patients. The cells were transfected with a mature microRNA-155 plasmid. The effect of microRNA-155 was assessed by Northern blotting, in situ hybridization, quantitative real-time PCR, immunofluorescent staining and Western blotting. In addition, activation of extracellular signal-regulated kinase $1 / 2$ was assessed by co-immunoprecipitation and Western blotting. Severely pre-eclamptic pregnant women expressed less mature miR-155 compared to healthy pregnant women. In addition, angiotensin II type 1 receptor expression decreased substantially in healthy cells and miR-155-transfected cells compared to miR-155-mutant-transfected cells and cells from pre-eclamptic patients. Mature miR-155 reduced angiotensin II-induced extracellular signal-regulated kinase $1 / 2$ activation. In conclusion, endogenous mature miR-155 expression may be an important contributor to the pathogenesis of severe pre-eclampsia.
\end{abstract}

\section{Introduction}

Pre-eclampsia, a serious disorder of pregnancy characterized by hypertension and proteinuria, is observed in approximately $5-7 \%$ of all pregnancies and results in 50,000-75,000 maternal deaths per year worldwide (1). Although several drugs are used in the clinical treatment of pre-eclampsia, the exact

Correspondence to: Dr Te Liu, School of Environmental Science and Engineering, Donghua University, Shanghai 201620, P.R. China E-mail: liute79@yahoo.com

Key words: angiotensin II, pre-eclampsia, extracellular signalrelated kinase $1 / 2$, miR-155, angiotensin II type 1 receptor pathogenesis of human pre-eclampsia is still unclear (2-5). Normal pregnancy is characterized by systemic inflammation, oxidative stress and alterations in levels of angiogenic factors and vascular reactivity. This is exacerbated in pre-eclampsia, with the associated breakdown of compensatory mechanisms, eventually leading to placental and vascular dysfunction (6).

Currently, angiotensin and angiogenic factors of the renin-angiotensin system are early important markers for preeclampsia (1,6-8). Angiotensin II (Ang II) has a critical role in the pathogenesis of pre-eclampsia. Ang II acts on endothelial cells, vascular smooth muscle cells and immune cells, accelerating pre-eclampsia at least partly by inducing low grade inflammation. Ang II stimulates vascular smooth muscle cells to produce monocyte chemoattractant protein, a major chemokine promoting macrophage infiltration. Macrophages, which accumulate in cardiac and other tissues, also produce angiotensin-converting enzyme, renin and Ang II (9-12). However, the molecular mechanisms by which regulation of hormone expression is involved in renin-angiotensin system function are unknown.

microRNAs (miRNAs), 20- to 23-nucleotide (nt) noncoding RNAs, function as sequence-specific gene expression regulators through translational repression and/or transcript cleavage (13-16). Since the roles and functions of endogenously expressed miRNAs were first described in C.elegans in 1993 (17), further work has shown that miRNAs have key roles in the regulation of development, especially the timing of morphogenesis and the maintenance of undifferentiated or incompletely differentiated cell types, such as stem cells $(18,19)$. Recent studies indicate that miRNA fragments can be captured and tested in cancer patient serum, and may be novel biomarkers for determining cancer stage and diagnosing hereditary diseases (20-22). Because of the complexity of the structure and clone processing of miRNAs, the assay and analysis requires large amounts of clinical tissue or blood samples.

microRNA-155 (miR-155) is overexpressed in a variety of human cancers and immune diseases (23-26). However, miR-155 is found at low levels in most immune cells until their activation by immune stimuli, such as antigens (27), 
Table I. Clinical characteristics of the pregnant women included in the study.

\begin{tabular}{lclc}
\hline Variable & Severe pre-eclamptic women $(\mathrm{n}=5)$ & Healthy pregnant women $(\mathrm{n}=5)$ \\
\hline Age (years) & $33 \pm 3$ & $(25-40)$ & $29 \pm 1 \quad(27-33)$ \\
Gestational age at sampling (days) & $255 \pm 13$ & $(212-282)$ & $265 \pm 2 \quad(255-269)$ \\
Systolic blood pressure $(\mathrm{mmHg})$ & $162.4 \pm 4.1$ & $(150-174)$ & $118.0 \pm 4.9(110-130)$ \\
Diastolic blood pressure $(\mathrm{mmHg})$ & $99.0 \pm 3.4$ & $(89-110)$ & $78.0 \pm 4.9(60-80)$ \\
Urine protein $(\mathrm{g} / 12 \mathrm{~h})$ & $4.45 \pm 0.35(3.78-5.39)$ & Negative \\
\hline
\end{tabular}

Toll-like receptor ligands, and inflammatory cytokines, which rapidly increase miR-155 expression (28). Additional studies have determined that miR-155 not only regulates tumor-related genes and immune cell function, but also the expression of renin-angiotensin system factors $(28,29)$. Elton et al (30) found that miR-155 regulated human Ang II type 1 receptor (AT1R) expression in fibroblast cells. In addition, the miR-155 on chromosome 21 differentially interacts with its polymorphic target in the AT1R 3' untranslated region (3'-UTR), which is the mechanism by which functional singlenucleotide polymorphisms regulate phenotypes (29).

However, whether miR-155 regulates AT1R expression in pre-eclampsia, and whether miR-155 is involved in preeclampsia pathogenesis, remains unknown. In this study, we isolated and cultured human umbilical vein endothelial cells (hUVECs) from severely pre-eclamptic patients and healthy puerperant women. The miR-155 expression vector was transfected into these cells to determine if miR-155 could specifically regulate AT1R. If miR-155 expression is involved in pre-eclampsia pathogenesis, it could be an important biomarker for this disease.

\section{Materials and methods}

Patients and control subjects. All samples were collected from the inpatient clinic of The International Peace Maternity and Child Health Hospital of The China Welfare Institute (Shanghai, China) between June 2008 and March 2010. All human materials were obtained with consent following approval by the Ethics Review Committee of the World Health Organization Collaborating Center for Research in Human Production authorized by the Shanghai Municipal Government. Due to material limitations, we could only analyze a limited number $(n=5)$ of severe pre-eclampsia cases. Healthy puerperant women $(n=5)$ were included for the controls. Clinical characteristics, such as age, blood pressure and urine protein, of the patients in this study are described in Table I.

Isolation and identification of $h U V E C s$. The human umbilical cords of all patients and healthy puerperant women were collected in asepsis after parturition. hUVECs were isolated by treatment with $1 \%$ trypsin, as previously described (31). hUVECs were grown on $1 \%$ gelatin-coated culture plates in McCoy's 5A medium (Sigma) supplemented with 15\% fetal bovine serum (Hyclone), $100 \mathrm{U} / \mathrm{ml}$ penicillin and $100 \mu \mathrm{g} / \mathrm{ml}$ streptomycin (Hyclone), at $37^{\circ} \mathrm{C}$ in a humidified atmosphere containing $5 \% \mathrm{CO}_{2}$. The cells were used within 2-3 passages and were identified as endothelial based on their cobblestone monolayer appearance in culture and by the expression of cell-surface antigens CD34 and CD31, as detected by immunohistochemistry.

Vector construction. For the expression plasmid pRNATCMV32-cGFP-mir155 (pre-miRNA of miR-155 expression element), oligonucleotide pairs for pre-miRNA of miR-155 and linker sequences with Bam $\mathrm{HI}$ and $\mathrm{XhoI}$ sites were chemically synthesized (23). The sequences of the oligonucleotides were: top strand, 5'-GTggatccCTGTTAATGCTAATCG TGATAGGGGTTTTTGCCTCCAACTGACTCCTACA TATTAGCATTAACAGctcgagCC-3', and bottom strand, 5'-GGctcgagCTGTTAATGCTAATATGTAGGAGTCAG TTGGAGGCAAAAACCCCTATCACGATTAGCATTA ACAGggatcc AC-3' (sequences corresponding to miR-155 seed sequences are capitalized, underlined and bold, and restriction enzyme sites are in lower case and underlined). To build the expression plasmid the oligonucleotide pairs were annealed and inserted into the multiple cloning sites between the BamHI and XhoI sites in the pRNAT-CMV32-cGFP/Neo vector (GenScript, NJ, USA). The negative control plasmid pRNAT-CMV32-cGFP-mir155-Mut was similarly built, except that 11 nucleotides in the sequence corresponding to the miR-155 seed sequence were mutated (change of TTAATGCTAAT to TaAtTcCaAtT; mutations are shown in lower case). Co-transfection of hUVECs from patients or healthy women were conducted to transfer $0.3 \mu \mathrm{g}$ miR-155 expression vector or miR-155 mutant vector, respectively, with Lipofectamine 2000 Reagent according to the manufacturer's protocol. The cells were seeded in a 6-well plate in McCoy 5A medium (Sigma-Aldrich) supplemented with $15 \%$ fetal bovine serum (Hyclone), $100 \mathrm{U} / \mathrm{ml}$ penicillin and $100 \mu \mathrm{g} / \mathrm{ml}$ streptomycin (Hyclone), at $37^{\circ} \mathrm{C}$ in a humidified atmosphere containing $5 \% \mathrm{CO}_{2}$, until $80 \%$ confluent.

$R N A$ extraction and analysis by quantitative real-time PCR $(q R T-P C R)$. Total RNA from the cells was isolated using TRIzol reagent (Invitrogen) according to the manufacturer's protocol. The RNA samples were treated with DNase I (Sigma-Aldrich), quantified, and reverse-transcribed into cDNA using the ReverTra Ace- $\alpha$ First Strand cDNA Synthesis kit (Toyobo). qRT-PCR was conducted using a RealPlex4 real-time PCR detection system from Eppendorf Co. Ltd. (Germany), with SYBR-Green Real Time PCR Master Mix (Toyobo) as the detection dye. qRT-PCR amplification was performed over 40 cycles with denaturation at $95^{\circ} \mathrm{C}$ for $15 \mathrm{sec}$ and annealing at $58^{\circ} \mathrm{C}$ for $45 \mathrm{sec}$. Target cDNA was quantified using the relative quantification method. A comparative 
threshold cycle $(\mathrm{Ct})$ was used to determine the gene expression relative to a control (calibrator). Steady-state mRNA levels are reported as an n-fold difference relative to the calibrator. For each sample, the $\mathrm{Ct}$ values of the genes were normalized using the formula $\Delta \mathrm{Ct}=\mathrm{Ct}_{\text {genes }}-\mathrm{Ct}_{18 \mathrm{sRNA}}$. To determine the relative expression levels, the following formula was used $\Delta \Delta \mathrm{Ct}=\Delta \mathrm{Ct}_{\text {all groups }}-\Delta \mathrm{Ct}_{\text {healthy group }}$. The values used to plot the relative expression of the markers were calculated using the expression $2^{-\Delta \Delta \mathrm{Ct}}$. The mRNA levels were calibrated based on the levels of 18s rRNA. The cDNA of each gene was amplified using primers as previously described $(9,32)$ : AT1R, forward: 5'-TCAGCCAGCGTCAGTTTCAA-3' and reverse: 5'-CTACAAGCATTGTGCGTCGAAG-3'; AT2R, forward: 5'-TTCCCTTCCATGTTCTGACC-3' and reverse: 5'-AAACACACTGCGGAGCTTCT-3'; ACE, forward: 5'-T GGCTCCTTCTCAGCCTTGTT-3' and reverse: 5'-AGGTC TTCGGCTTCGTGGTTA-3'; and Ang II, forward: 5'-ACGT CCACTTCCAAGGGAAGA-3' and reverse: 5'-CGAGAAGT TGTCCTGGATGTCAC-3'.

Northern blotting analysis. All steps of Northern blotting were performed as previously described (33). For all groups, $20 \mu \mathrm{g}$ of good quality total RNA were analyzed on a 7.5 M ureum $12 \%$ PAA denaturing gel and transferred to a Hybond $\mathrm{N}^{+}$nylon membrane (Amersham, Freiburg, Germany). Membranes were cross-linked using UV light for $30 \mathrm{sec}$ at $1200 \mathrm{~mJ} / \mathrm{cm}^{2}$. Hybridization was performed with the miR-155 antisense starfire probe, 5'-CCCCTATCACGATTAGCATTAA-3' (IDT, Coralville, IA), to detect the 22-nt miR-155 fragments according to the manufacturer's instructions. After washing, membranes were exposed for 20-40 h to Kodak XAR-5 film (SigmaAldrich Chemical). As a positive control, all membranes were hybridized with a human U6 snRNA probe, 5'-GCAGGGG CCATGCTAATCTTCTCTGTATCG-3'. Exposure times for the U6 control probe varied from 15-30 min.

RNA in situ hybridization. RNA in situ hybridization was used to detect endogenous miR-155 expression on the hUVECs of between severe pre-eclampsia and healthy puerperant women (5 cases each) as previously described $(33,34)$. Briefly, cells were deparaffinized and air-dried for $10 \mathrm{~min}$. Slides were treated with proteinase $\mathrm{K}$ (Roche) at $37^{\circ} \mathrm{C}$ for $3 \mathrm{~min}$. Generally, four different concentrations of proteinase $\mathrm{K}$ were used $(5,10,15$, and $20 \mu \mathrm{g} / \mathrm{ml}$ in TBS) for each cell sample. After being washed with PBS, slides were incubated with $1 \mathrm{ng} / \mu 1$ DIG-labeled probe (antisense or sense) in a hybridization solution consisting of 5X Denhardt's solution, 2X SSC, $10 \%$ dextran, $30 \%$ formamide, $1 \mathrm{mg} / \mathrm{ml} \mathrm{t}-\mathrm{RNA}$, and $2 \mathrm{mg} / \mathrm{ml}$ fish sperm DNA overnight at $42^{\circ} \mathrm{C}$ after being washed. Positive cells were visualized with anti-DIG-labeled alkaline phosphatase (Roche) for $1 \mathrm{~h}$ in $0.1 \mathrm{M}$ maleic acid buffer containing $0.15 \mathrm{M} \mathrm{NaCl}, 2 \%$ blocking buffer, and $1 \%$ Triton X-100. The staining reaction was performed overnight with NBT and BCIP (Roche) in TBS buffer ( $\mathrm{pH}$ 9.0) containing $50 \mathrm{mM} \mathrm{MgCl}$ and $0.01 \mathrm{M}$ levamisole. All cases were routinely stained with a probe for U6 as a positive control for the RNA quality and fixation of the cell samples.

Luciferase reporter assay. All the steps were performed as previously described $(35,36)$. NIH-3T3 cells were seeded at
$3 \times 10^{4} /$ well in 48 -well plates and co-transfected with $400 \mathrm{ng}$ of pRNAT-CMV32-cGFP-mir155 or pRNAT-CMV32-cGFP or pRNAT-CMV32-cGFP-mir155-Mut, $20 \mathrm{ng}$ of pGL3cmAT1R-3UTR-WT or pGL3cm-AT1R-3UTR-Mut, and pRL-TK (Promega, Madison, USA) using the Lipofectamine 2000 Reagent according to the manufacturer's protocol. After $48 \mathrm{~h}$ transfection, luciferase activity was measured using the dualluciferase reporter assay system (Promega).

Immunofluorescence staining analysis. The cultured cells were washed 3 times with PBS and fixed with $4 \%$ paraformaldehyde (Sigma-Aldrich, St. Louis, USA) for $30 \mathrm{~min}$. After blocking, the cells were incubated first with rabbit anti-human Ang II polyclonal antibody (1:200; Santa Cruz Biotechnologhy Inc., Santa Cruz, USA) and rabbit anti-human AT1R polyclonal antibody (1:200; Santa Cruz Biotechnology Inc.) overnight at $4{ }^{\circ} \mathrm{C}$, and then with $\mathrm{Cy} 3$-conjugated goat anti-rabbit IgG antibody (1:200; Abcam, Cambridge, UK) and $5 \mathrm{mg} / \mathrm{ml}$ DAPI (Sigma-Aldrich) at room temperature for $30 \mathrm{~min}$. Then the cells were thoroughly washed with TBST and viewed with a fluorescence microscope (DMI3000; Leica, Allendale, NJ, USA).

Co-immunoprecipitation assays. The cell groups were seeded at $3 \times 10^{5}$ per well in 6 -well plates and cultured until $85 \%$ confluent, then lysed (500 $\mu 1$ per plate) in a modified cell lysis buffer for Western blot and IP analysis (20 mM Tris, $\mathrm{pH} 7.5$; $150 \mathrm{mM} \mathrm{NaCl}, 1 \%$ Triton X-100, 1 mM EDTA, sodium pyrophosphate, $\beta$-glycerophosphate, $\mathrm{Na}_{3} \mathrm{VO}_{4}$ and leupeptin) (Beyotime Institute of Biotechnology). After lysis, each sample was centrifuged to clear the lysate of the insoluble debris and preincubated with $20 \mu \mathrm{g}$ protein A agarose beads (Beyotime Institute of Biotechnology) by rocking for $30 \mathrm{~min}$ at $4{ }^{\circ} \mathrm{C}$, followed by centrifugation and transfer to a fresh $1.5 \mathrm{ml}$ tube. The rabbit anti-human extracellular signal regulated kinase (ERK) $1 / 2$ polyclonal antibody (Cell Signaling Technology, USA) was incubated for 90 min before a second addition of $20 \mu \mathrm{g}$ protein A agarose beads to capture the immune complexes. The pelleted beads were then washed three times with $500 \mu \mathrm{l}$ cell lysis buffer, dissolved in 4X SDS-PAGE sample loading buffer, and heated for $10 \mathrm{~min}$ at $95^{\circ} \mathrm{C}$.

Western blotting analysis. Total protein extracts of each group of cells were resolved by $12 \%$ SDS-PAGE and transferred to PVDF (Millipore) membranes. After blocking, the PVDF membranes were washed 4 times for 15 min with TBST at room temperature and incubated with the following primary antibodies: rabbit anti-human Ang II polyclonal antibody (1:500; Santa Cruz Biotechnologhy Inc.); rabbit anti-human ACE polyclonal antibody (1:500; Santa Cruz Biotechnology Inc.); rabbit anti-human AT1R polyclonal antibody (1:500; Santa Cruz Biotechnology Inc.); rabbit anti-human AT2R polyclonal antibody (1:500; Santa Cruz Biotechnology Inc.); and rabbit anti-human ERK1/2 and pERK1/2 polyclonal antibody (1:1000; Cell Signaling Technology). Following extensive washing, the membranes were incubated with a secondary peroxidase-linked goat anti-rabbit IgG (1:1000, Santa Cruz) for $1 \mathrm{~h}$. After washing 4 times for $15 \mathrm{~min}$ with TBST at room temperature once more, the immunoreactivity was visualized by enhanced chemiluminescence (ECL kit, 
A.

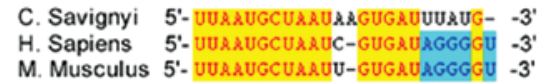

B.

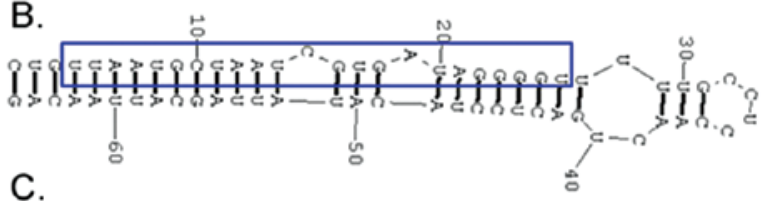

Figure 1. The human AT1R mRNA 3'-UTR harbors miR-155 binding sites and the secondary structure of miR-155. (A) The mature miR-155 RNA sequences of multi-species were analyzed and contrasted by bioinformatic tools. (B) The typical secondary structure of precursor microRNAs (pre-miRNAs) with hsa-miR-155. There are some stem-loop and hairpin structures in the pre-miRNAs. The common binding site is located in an unstable region with a multi-branching loop-like RNA structure. The pane indicates the mature miRNAs which were bound with the 3'-UTR of the target genes. (C) Complementarity between miR-155 and the putative human AT1R 3'-UTR targeted site (70-90 bp downstream). Also shown are the conserved bases of the putative miR-155 target sequence present in the human AT1R 3'-UTR.

Pierce Biotechnology), and membranes were exposed to Kodak XAR-5 film (Sigma-Aldrich).

Statistical analysis. Each experiment was performed as least three times, and data are shown as the mean $\pm \mathrm{SE}$ where applicable. Differences were evaluated using the Student's t-test. The probability of $<0.05$ was considered to be statistically significant.

\section{Results}

Bioinformatics analysis of miR-155 and of the 3'-UTR of $A T I R$ mRNA. The pre-miRNA sequences, mature miRNA sequences, chromosomal locations and length of miR-155 and the target gene AT1R in multi-species were analyzed using the online research tools of the miRBase Target database (http://www.mirbase.org) $(28,33)$. Nine to 16 putative miRNA target sites were harbored in the 3'-UTR of AT1R mRNA, depending on the species analyzed. For this study, we focused on the human miR-155, which may target the human AT1R 3'-UTR, since these sites are conserved, to varying degrees, across species (Fig. 1).

Exogenous miR-155 expressed in severely pre-eclamptic pregnant women hUVECs interfered with ATIR expression. In situ hybridization revealed a great number of strongly stained hUVECs in samples from healthy pregnant women, but not from severely pre-eclamptic pregnant women (Fig. 2). In addition, Northern blotting revealed that the mature miR-155 signal in hUVECs from severely pre-eclamptic pregnant women was weaker than in hUVECs from healthy pregnant women. However, mature miR-155 was only found in healthy hUVECs (Fig. 3). Finally, exogenous miR-155 was expressed in the hUVECs after transfection with the miR-155 expressed plasmid, but not in hUVECs transfected with the mutant miR-155 plasmid.

Plasmid DNA of each AT1R mRNA 3'-UTR site [wildtype (WT) AT1R, empty plasmid, and mutant AT1R] were co-transfected with miR-155 expression vector (WT miR-155, empty vector, and mutant miR-155) into NIH-3T3 cells to examine whether the AT1R gene expression was regulated by mature miR-155. Luciferase activity of the AT1R 3'-UTR sites was inhibited significantly by WT miR-155 (Fig. 3), while

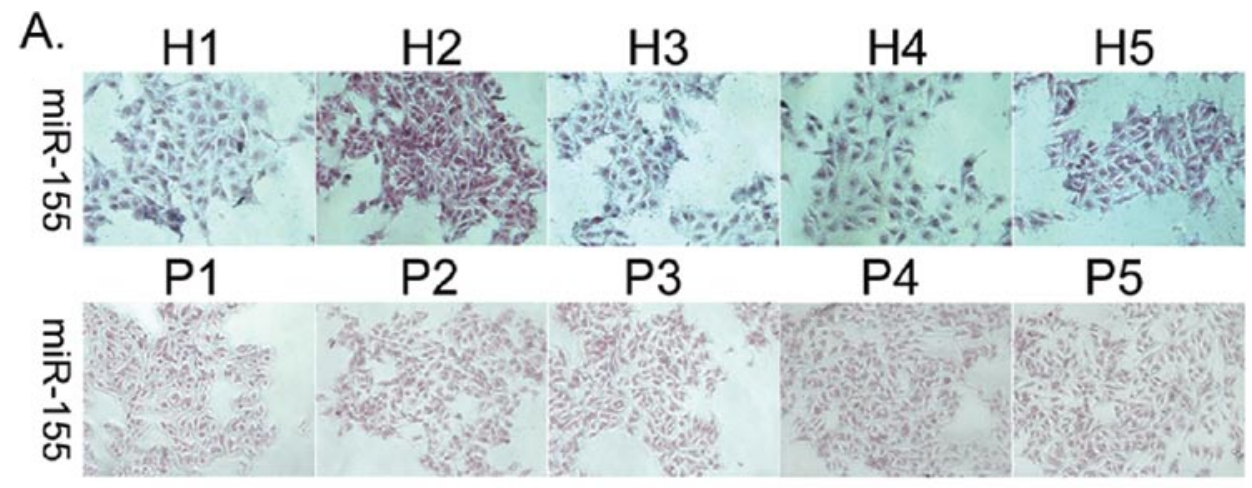

B.

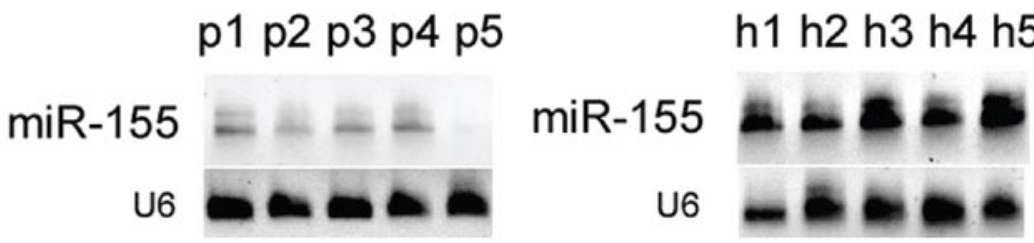

Figure 2. The in situ hybridization and Northern blotting assays to identify the mature miR-155 expression specifically by the mature miR-155 probe. (A) The results of in situ hybridization show that a great number of hUVECs of healthy pregnant women, but not of severe pre-eclampsia pregnant women are stained deep purple. $\mathrm{H} 1$ to $\mathrm{H} 5$ are the 5 cases of healthy pregnant women; P1 to P5 are the 5 cases of severe pre-eclampsia pregnant women. Original magnification x100. (B) The results of Northern blotting show that the hybridized signal of the mature miR-155 in hUVECs of severe pre-eclampsia pregnant women was weaker than that of healthy pregnant women. h1 to h5 are the 5 cases of healthy pregnant women; p1 to p5 are the 5 cases of severe pre-eclampsia pregnant women. The human U6 probe was used as a loading control. 


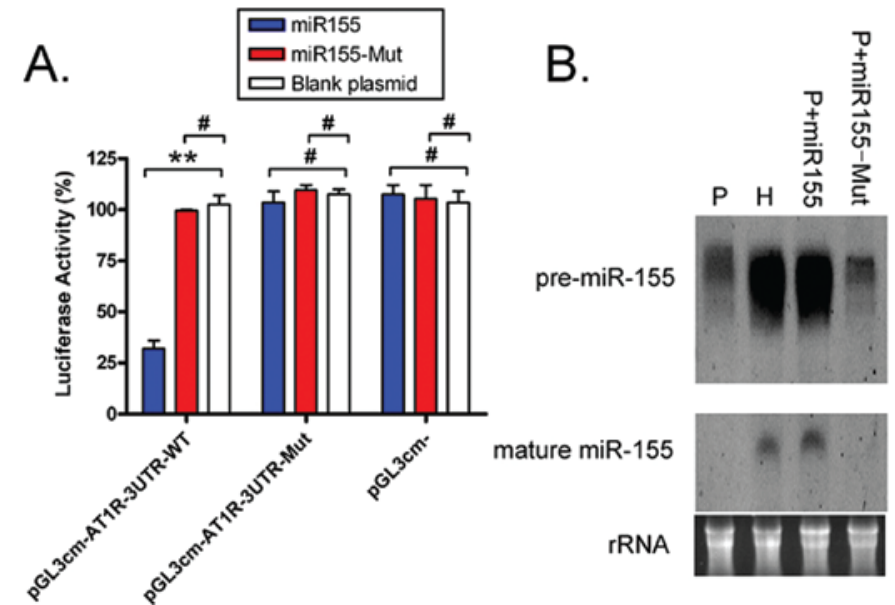

Figure 3. Luciferase and Northern blot assays to determine the miRNA-155 expression and effect on the target gene AT1R in different groups. (A) WT reporter or mutated control luciferase plasmids were transfected into NIH-3T3 cells with miR-155 expressed plasmids or miR-155 mutant expression plasmids. After the assay, the luciferase activity with the AT1R $3^{\prime} \mathrm{UTR}$ sites was significantly inhibited by miR-155. ${ }^{* *} \mathrm{P}<0.01 \mathrm{vs}$. blank plasmid; ${ }^{\sharp} \mathrm{P}>0.05 \mathrm{vs}$. blank plasmid; $n=5$. (B) Northern blotting experiment showing exogenous and endogenous miR-155 expression in the different groups. rRNA was used as a loading control. $\mathrm{H}$, healthy pregnant women group; P, pre-eclampsia pregnant women group; P+miR155, miR-155 expression plasmid transfected into the hUVECs of pre-eclampsia pregnant women; P+miR155-Mut, miR-155 mutant expression plasmid transfected into the hUVECs of pre-eclampsia pregnant women.

luciferase activity of the mutated AT1R 3'-UTR sites was not inhibited. These data suggest that miR-155 targets AT1R.

miR-155 specifically influences the expression of reninangiotensin system factors. To determine whether exogenous and endogenous miR-155 could influence the expression of renin-angiotensin system factors (AT1R, AT2R, angiotensin-converting enzyme and Ang II), expression of these factors was assessed by immunofluorescence (IF) staining, qRT-PCR and Western blotting. AT1R expression decreased in healthy cells and miR-155-transfected cells compared to pre-eclamptic patient cells and mutant-miR-155-transfected cells (Fig. 4). However, Ang II expression was not remarkably different between the four groups. These results suggest that not only was the expression of renin-angiotensin factors increased in hUVECs from severely pre-eclamptic pregnant women, but also miRNA-155 expression only influenced endogenous AT1R expression but not that of other reninangiotensin factors.

The qRT-PCR results confirmed the IF results. When the miR-155 expression vector was transfected into the cells, AT1R expression, but not that of other renin-angiotensin factors, decreased compared to untransfected cells or miR-155 mutant transfected cells, Relative mRNA expression is shown after normalization to $18 \mathrm{~s}$ rRNA, which served as an internal control (Fig. 3). Interestingly, Western blotting revealed that AT1R levels in cells from pre-eclampsia patients or miR-155mutant transfected cells were $0.729 \pm 0.042$ and $0.642 \pm 0.059$ of $\beta$-actin expression, respectively (Fig. 5). These values were significantly higher than those for the miR-155-transfected group or healthy group $(0.265 \pm 0.025$ and $0.167 \pm 0.027$ of $\beta$-actin, respectively). These data indicate that exogenous and endogenous miR-155 down-regulates AT1R expression, but not that of other renin-angiotensin system factors (AT2R, angiotensin-converting enzyme and Ang II).

miR-155 reduced Ang II-induced ERK1/2 activation. To investigate whether miR-155 reduces ERK1/2 phosphorylation

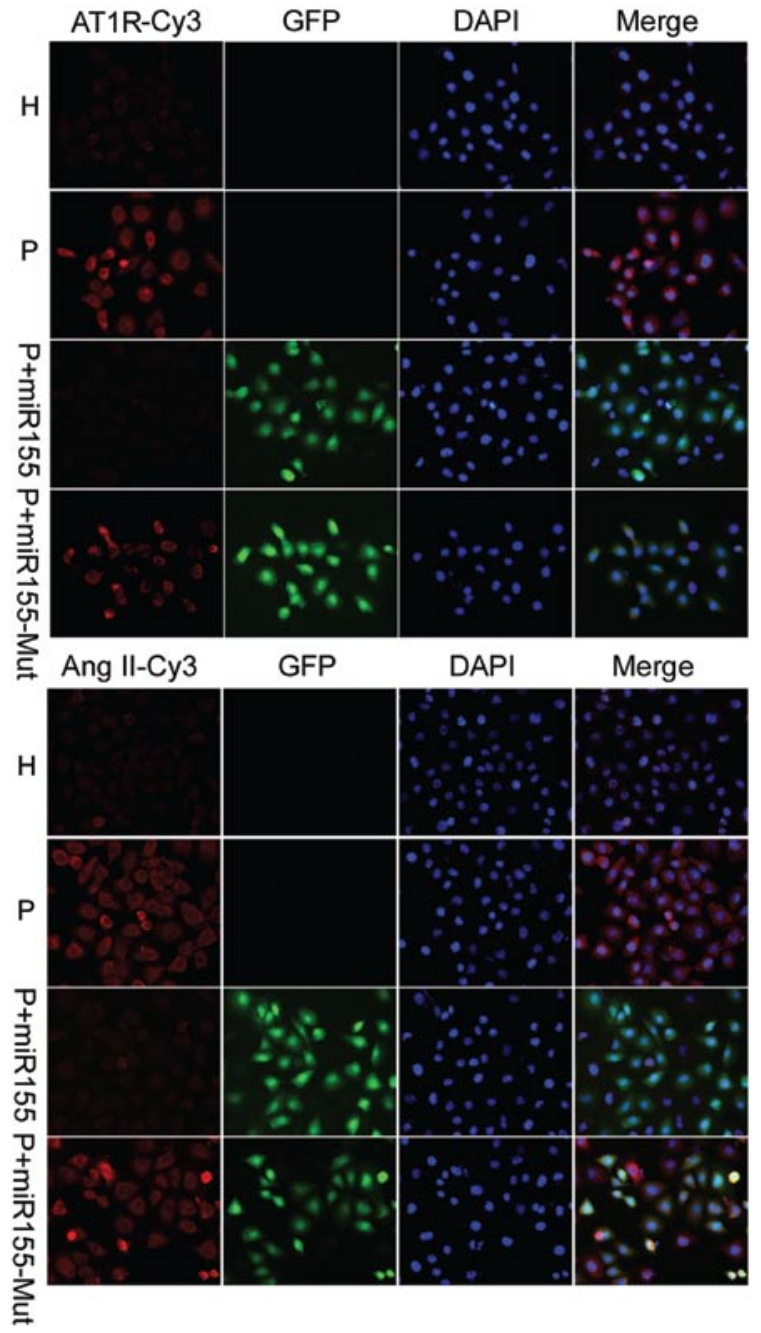

Figure 4. Immunofluorescence (IF) staining to determine whether exogenous and endogenous miR-155 could influence the expression of renin-angiotensin system factors. $\mathrm{H}$, healthy pregnant women group; $\mathrm{P}$, pre-eclampsia pregnant women group; $\mathrm{P}+\mathrm{miR} 155$, miR-155 expression plasmid transfected into the hUVECs of pre-eclampsia pregnant women; P+miR155-Mut, miR-155 mutant expression plasmid transfected into the hUVECs of pre-eclampsia pregnant women. 


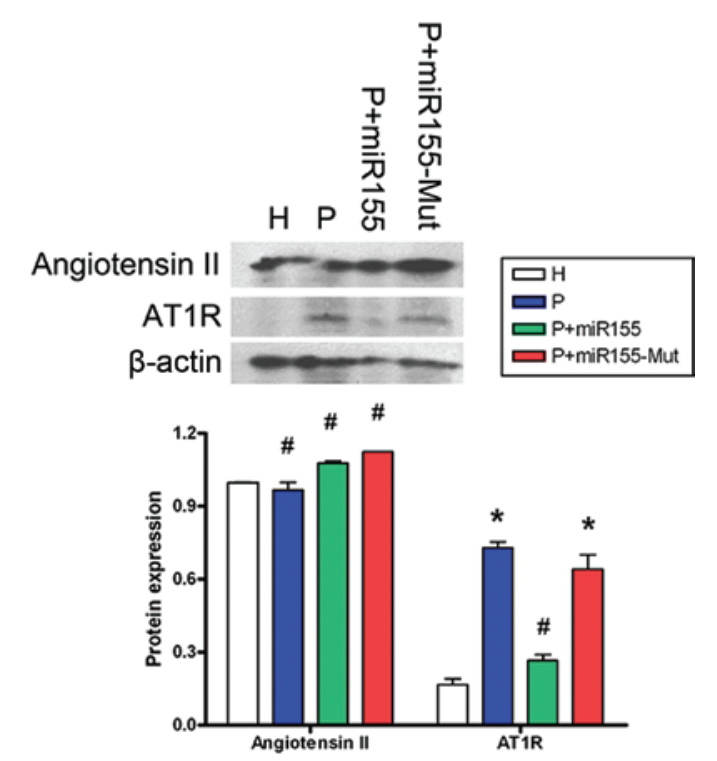

Figure 5. Western blot assay to determine the expression of Angiotensin II and AT1R in the different groups. The results demonstrate that AT1R levels in cells from pre-eclampsia patients or miR-155-mutant transfected cells are significantly higher than those of the miR-155-transfected or the healthy groups. The data indicate that exogenous and endogenous miR-155 down-regulates AT1R expression, but not that of other renin-angiotensin system factors. $\mathrm{H}$, healthy pregnant women group; $\mathrm{P}$, pre-eclampsia pregnant women group; $\mathrm{P}+\mathrm{miR} 155$, miR-155 expression plasmid transfected into the hUVECs of pre-eclampsia pregnant women; P+miR155-Mut, miR-155 mutant expression plasmid transfected into the hUVECs of pre-eclampsia pregnant women. ${ }^{*} \mathrm{P}<0.05$ vs. the healthy pregnant women group, $\mathrm{H}$; ${ }^{"} \mathrm{P}>0.05$ vs. the healthy pregnant women group; $\mathrm{n}=5$.

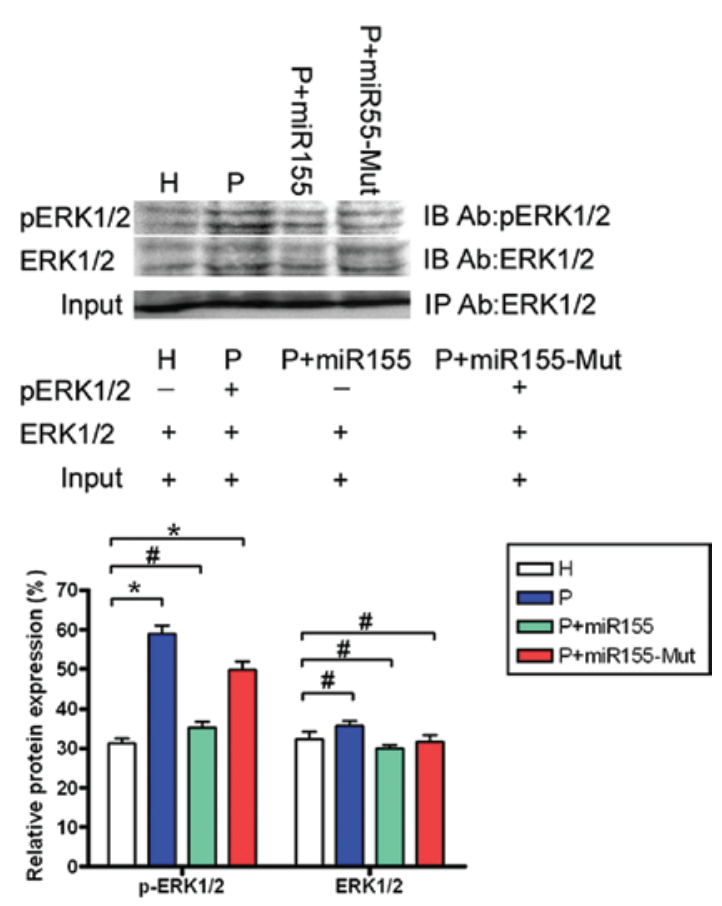

Figure 6. The co-IP Western blotting experiment to determine the miRNA-155 expression and the influence on the angiotensin II-induced extracellular signal related kinase 1/2 (ERK1/2) activation. Levels of phospho-ERK1/2 in patient group or miR-155-mutant transfected group were significantly higher than those for the miR-155-transfected group or healthy group. Input was used as a loading control. $\mathrm{H}$, healthy pregnant women group; $\mathrm{P}$, pre-eclampsia pregnant women group; $\mathrm{P}+\mathrm{miR} 155$, miR-155 expression plasmid transfected into the hUVECs of pre-eclampsia pregnant women; P+miR155-Mut, miR-155 mutant expression plasmid transfected into the hUVECs of pre-eclampsia pregnant women. ${ }^{\mathrm{P}}<0.05$ vs. Blank plasmid; ${ }^{\mathrm{P}} \mathrm{P}>0.05$ vs. Blank plasmid; $\mathrm{n}=5$. and activation in hUVECs from severely pre-eclamptic pregnant women, phospho-ERK $1 / 2$ was detected by coimmunoprecipitation followed by Western blotting. The phospho-ERK1/2 levels in cells from pre-eclampsia patients or miR-155-mutant transfected cells were $58.95 \pm 3.64 \%$ or $49.81 \pm 3.72 \%$ of input expression levels, respectively. These values were significantly higher than those for the miR-155transfected cells or cells from healthy puerperant women (29.88 $\pm 1.58 \%$ or $32.38 \pm 3.24 \%$ of input levels, respectively). However, there were no obviously difference of the levels of normal ERK1/2 expression in each groups (Fig. 6). These results show that miR-155, both endogenous and exogenous, significantly reduced Ang II-induced ERK1/2 activation.

\section{Discussion}

Increasing evidence shows that miR-155 regulates the expression of AT1R in the renin-angiotensin system $(28,29)$ Down-regulation or deletion of miR-155 leads to overexpression of AT1R, suggesting AT1R is a target gene of miR-155. Our preliminary experiments demonstrated that reninangiotensin system expressions, especially Ang II and AT1R, were significantly increased in hUVECs from patients with early-onset severe pre-eclampsia. Based on these findings, we speculate severe pre-eclampsia is possibly correlated with the aberrant expression or loss of miR-155. In this study, we showed that hUVECs from severely pre-eclamptic pregnant women expressed less mature miR-155 than hUVECs from healthy pregnant women. In addition, miR-155 targeted AT1R and reduced Ang II-induced ERK1/2 phosphorylation and activation. In contrast to a previous report $(28,29)$ that exogenous miR-155 expression in human fibroblasts inhibited endogenous AT1R protein expression, we found that AT1R expression was decreased in cells from healthy puerperant women and miR-155-transfected cells compared to cells from pre-eclampsia patients and mutant-miR-155-transfected cells. However, Ang II expression was not significantly altered. These results suggest that not only did the expression of renin-angiotensin factors increase in hUVECs from severely pre-eclamptic pregnant women, but also that miRNA-155 expression influenced endogenous AT1R expression, but not the expression of other renin-angiotensin factors. Taken together, these results also suggest that the development of pre-eclampsia is possibly related to miR-155 expression. The inhibition or loss of miR-155 expression can result in excessive AT1R and thus probably influence renin-angiotensin system expression, which may contribute to the development of pre-eclampsia.

\section{Acknowledgements}

This study was supported by grants from the Shanghai Municipal Health Bureau Fund for Young Scholars (No. 2008Y002), Medicine-Engineering Unite Fund for Shanghai Jiaotong University (No. YG2009MS47), Natural Science Foundation of School of Medicine of Shanghai Jiaotong University and Shanghai Committee Medical Science Foundation of China (No. 10411967100) to T.L. This study was also supported by a grant from National Key Technology R\&D Program (No. 2006BAI05A05) to W.C. 


\section{References}

1. Steinberg G, Khankin EV and Karumanchi SA: Angiogenic factors and preeclampsia. Thromb Res 123 (Suppl. 2): S93-S99, 2009.

2. Mounier-Vehier C and Delsart P: Pregnancy-related hypertension: a cardiovascular risk situation. Presse Med 38: 600-608, 2009 (In French).

3. Thangaratinam S, Coomarasamy A, O'Mahony F, et al: Estimation of proteinuria as a predictor of complications of pre-eclampsia: a systematic review. BMC Med 7: 10, 2009.

4. Hofmeyr GJ and Belfort M: Proteinuria as a predictor of complications of pre-eclampsia. BMC Med 7: 11, 2009.

5. Scalera F, Fischer T, Schlembach D and Beinder E: Serum from healthy pregnant women reduces oxidative stress in human umbilical vein endothelial cells. Clin Sci (Lond) 103: 53-57, 2002.

6. Myatt L and Webster RP: Vascular biology of preeclampsia. J Thromb Haemost 7: 375-384, 2009.

7. Stepan H: Angiogenic factors and pre-eclampsia: an early marker is needed. Clin Sci (Lond) 116: 231-232, 2009.

8. Cudihy D and Lee RV: The pathophysiology of pre-eclampsia: current clinical concepts. J Obstet Gynaecol 29: 576-582, 2009.

9. Tone A, Shikata K, Ogawa D, et al: Changes of gene expression profiles in macrophages stimulated by angiotensin II - angiotensin II induces MCP-2 through AT1-receptor. J Renin Angiotensin Aldosterone Syst 8: 45-50, 2007.

10. Dzau VJ: Vascular renin-angiotensin system and vascular protection. J Cardiovasc Pharmacol 22 (Suppl. 5): S1-S9, 1993.

11. Diet F, Pratt RE, Berry GJ, Momose N, Gibbons GH and Dzau VJ: Increased accumulation of tissue ACE in human atherosclerotic coronary artery disease. Circulation 94: 2756-2767, 1996.

12. Chen XL, Tummala PE, Olbrych MT, Alexander RW and Medford RM: Angiotensin II induces monocyte chemoattractant protein-1 gene expression in rat vascular smooth muscle cells. Circ Res 83: 952-959, 1998

13. Luzi E, Marini F, Sala SC, Tognarini I, Galli G and Brandi ML: Osteogenic differentiation of human adipose tissue-derived stem cells is modulated by the miR-26a targeting of the SMAD1 transcription factor. J Bone Miner Res 23: 287-295, 2008.

14. Zhang J, Du YY, Lin YF, et al: The cell growth suppressor, mir-126, targets IRS-1. Biochem Biophys Res Commun 377: 136-140, 2008

15. Yu B, Chapman EJ, Yang Z, Carrington JC and Chen X: Transgenically expressed viral RNA silencing suppressors interfere with microRNA methylation in Arabidopsis. FEBS Lett 580: 3117-3120, 2006

16. Bartel DP: microRNAs: genomics, biogenesis, mechanism, and function. Cell 116: 281-297, 2004

17. Lee RC, Feinbaum RL and Ambros V: The C. elegans heterochronic gene lin-4 encodes small RNAs with antisense complementarity to lin-14. Cell 75: 843-854, 1993.

18. Carrington JC and Ambros V: Role of microRNAs in plant and animal development. Science 301: 336-338, 2003.

19. Wang Y, Keys DN, Au-Young JK and Chen C: MicroRNAs in embryonic stem cells. J Cell Physiol 218: 251-255, 2009.
20. Chen X, Ba Y, Ma L, et al: Characterization of microRNAs in serum: a novel class of biomarkers for diagnosis of cancer and other diseases. Cell Res 18: 997-1006, 2008.

21. Mitchell PS, Parkin RK, Kroh EM, et al: Circulating microRNAs as stable blood-based markers for cancer detection. Proc Natl Acad Sci USA 105: 10513-10518, 2008.

22. Gilad S, Meiri E, Yogev Y, et al: Serum microRNAs are promising novel biomarkers. PLoS One 3: e3148, 2008.

23. Jiang S, Zhang HW, Lu MH, et al: MicroRNA-155 functions as an OncomiR in breast cancer by targeting the suppressor of cytokine signaling 1 gene. Cancer Res 70: 3119-3127, 2010.

24. Eis PS, Tam W, Sun L, et al: Accumulation of miR-155 and BIC RNA in human B cell lymphomas. Proc Natl Acad Sci USA 102: 3627-3632, 2005.

25. Wu S, Huang S, Ding J, et al: Multiple microRNAs modulate p21Cip1/Waf1 expression by directly targeting its 3' untranslated region. Oncogene 29: 2302-2308, 2010.

26. Landgraf $\mathrm{P}$, Rusu M, Sheridan R, et al: A mammalian microRNA expression atlas based on small RNA library sequencing. Cell 129: 1401-1414, 2007.

27. Teng G and Papavasiliou FN: Shhh! Silencing by microRNA-155. Philos Trans R Soc Lond B Biol Sci 364: 631-637, 2009.

28. Martin MM, Lee EJ, Buckenberger JA, Schmittgen TD and Elton TS: MicroRNA-155 regulates human angiotensin II type 1 receptor expression in fibroblasts. J Biol Chem 281: 18277-18284, 2006.

29. Sethupathy P, Borel C, Gagnebin M, et al: Human microRNA-155 on chromosome 21 differentially interacts with its polymorphic target in the AGTR1 3' untranslated region: a mechanism for functional single-nucleotide polymorphisms related to phenotypes. Am J Hum Genet 81: 405-413, 2007.

30. Martin MM, Buckenberger JA, Jiang J, et al: The human angiotensin II type 1 receptor $+1166 \mathrm{~A} / \mathrm{C}$ polymorphism attenuates microrna-155 binding. J Biol Chem 282: 24262-24269, 2007.

31. Ko Y, Totzke G, Seewald S, et al: Native low-density lipoprotein (LDL) induces the expression of the early growth response gene-1 in human umbilical arterial endothelial cells. Eur J Cell Biol 68: 306-312, 1995.

32. Uemura $H$, Hasumi $H$, Ishiguro $H$, Teranishi J, Miyoshi $Y$ and Kubota Y: Renin-angiotensin system is an important factor in hormone refractory prostate cancer. Prostate 66: 822-830, 2006.

33. Kluiver J, Haralambieva E, de Jong D, et al:: Lack of BIC and microRNA miR-155 expression in primary cases of Burkitt lymphoma. Genes Chromosomes Cancer 45: 147-153, 2006.

34. van den Berg A, Kroesen BJ, Kooistra K, et al: High expression of B-cell receptor inducible gene BIC in all subtypes of Hodgkin lymphoma. Genes Chromosomes Cancer 37: 20-28, 2003.

35. He M, Xu Z, Ding T, Kuang DM and Zheng L: MicroRNA-155 regulates inflammatory cytokine production in tumor-associated macrophages via targeting C/EBPbeta. Cell Mol Immunol 6: 343-352, 2009

36. Huang S, He X, Ding J, et al: Upregulation of miR-23a approximately 27 a approximately 24 decreases transforming growth factor- $\beta$-induced tumor-suppressive activities in human hepatocellular carcinoma cells. Int J Cancer 123: 972-978, 2008. 